\title{
Enseñar o aprender periodismo. El modelo pedagógico de la Fundación García Márquez para el Nuevo Periodismo Iberoamericano (FNPI)
}

\author{
Miguel Ángel DEL ARCo BRAVo \\ Universidad Carlos III de Madrid \\ miguelangel.arco@uc3m.es
}

Recibido: 14 de diciembre de 2014

Aceptado: 12 de mayo de 2015

\begin{abstract}
Resumen
La profesión periodística pasa por un mal momento, la precariedad y la falta de credibilidad parecen haberse instalado en ella. Las causas de esa situación están relacionadas directamente con la crisis económica y las dudas empresariales del modelo de negocio ante las nuevas tecnologías, pero también con el abandono o incumplimiento de algunas reglas sagradas, como el rigor, la ética y la calidad. Esas cualidades esenciales, ¿se pueden aprender?, ¿es posible enseñarlas? Los medios y los empresarios desconfían de los estudios reglados y acusan a las universidades de crear periodistas que no salen preparados. La academia denuncia un intento de denostar la preparación universitaria en pos de mano de obra barata y periodistas maleables. Este artículo estudia el caso de la Fundación Nuevo Periodismo Iberoamericano (FNPI), una institución de referencia mundial ideada y creada por Gabriel García Márquez en 1995 precisamente con la intención de recuperar elementos fundamentales del periodismo. Lleva veinte años formando a jóvenes periodistas sobre la base de fomentar la capacidad de narrar, la investigación rigurosa y la ética. Un modelo pedagógico propio que propone un camino para la recuperación de los valores y la consideración del periodismo. El ejemplo de los maestros de prestigio parece indispensable en cualquier aprendizaje.
\end{abstract}

Palabras clave: Periodismo, investigación, ética, calidad, Iberoamérica, pedagogía, universidad, impacto.

\section{To teach or to learn journalism. The pedagogical model of the García Márquez foundation for a new ibero-american journalism}

\begin{abstract}
Journalism is traversing a bad period: precarity and lack of credibility appear nowadays as integral to the profession. The reasons for this situation are directly related to the economic crisis and managerial doubts regarding a business model faced with new technologies. But the causes can also be traced back to the abandonment or failure to comply with sacred norms such as rigor, ethics and quality. Can these defining and moral qualities be learned? Is it possible to teach them? Media and business owners do not trust formal education and accuse universities of creating unprepared journalists. The academic environment denounces the attempt to disparage university training as part of the search for cheap labour and compliant journalists. This article analyzes the case of the Foundation for a New Ibero-American Journalism. The Foundation is an institution of worldwide reputation, designed and set up by Gabriel García Márquez in 1995 with precisely the purpose of recovering essential elements of journalism. For 20 years it has been training young journalists on the basis of developing strong narrative skills, a commitment to rigorous investigation and ethical responsibility. A pedagogical model that puts forward a model for reclaiming journalism's prestige, principles and public status. The example of the masters appears indispensable to all forms of learning.

Keywords: Journalism, research, ethics, quality, Latin America, teaching, university, public recognition.

\section{Referencia normalizada}

DEL ARCO BRAVO, Miguel Ángel (2015): "Enseñar o aprender periodismo. El modelo pedagógico de la Fundación García Márquez para el Nuevo Periodismo Iberoamericano (FNPI)". Estudios sobre el Mensaje Periodístico. Vol. 21, Núm. 2 (julio-diciembre), págs.: 1031-1044. Madrid, Servicio de Publicaciones de la Universidad Complutense.
\end{abstract}


Sumario: 1. Introducción. 2. Propósito y metodología. 3. Caso de estudio. 4. Los logros. 5. Discusión. 6. Conclusión. 7. Referencias bibliográficas.

\section{Introducción}

Lo que es hoy la FNPI, un referente internacional en el periodismo de calidad, un lugar donde los mejores periodistas del continente aprenden de la mano de los principales maestros del mundo, parte de un sueño que tuvo García Márquez hace 20 años. En 1994 le rondó la idea de hacer algo ante la deriva que iba tomando el periodismo: Notó su desprestigio y abandono, percibió el peligro de que los medios hicieran depender su éxito de la primicia y de la lucha por la inmediatez, caviló sobre lo bueno y lo malo de las nuevas tecnologías... Y tales preocupaciones las compartió con amigos y periodistas, con Tomás Eloy Martínez, Alma Guillermoprieto, Jaime Abello. Entre todos pensaron un método pedagógico para poner remedio y además buscaron la financiación para llevarlo a cabo.

El sueño se fue convirtiendo en un ambicioso proyecto educativo internacional enfocado a la reflexión sobre el periodismo, la experimentación de laboratorio, a la formación de los profesionales y a la calidad de las prácticas periodísticas, eso en el contexto de una época de transición de medios impresos y audiovisuales tradicionales a medios basados en la convergencia digital.

Desde entonces miles de personas han estudiado o enseñado en la Fundación, participando en cientos de actividades. Se ha conformado la mejor cantera de periodistas de América Latina y la más sobresaliente selección de maestros.

Pero la fantasía de visionario no fue enteramente casual porque García Márquez había tenido antes profundas preocupaciones periodísticas y pedagógicas. Practicó el periodismo en su juventud, como columnista, cronista, redactor y reportero. Volvió en varias ocasiones, como cuando decidió escribir Noticia de un secuestro (1991), un hibrido entre la crónica y la novela ${ }^{1}$, o cuando fue inversor en la revista Cambio (1993). Además había escrito, y ensayado el modelo educativo de talleres prácticos basados en las vocaciones en sus textos "Manual para ser niño" y "Por un país al alcance de los niños"2.

De modo que las carencias y las necesidades del periodismo y de la escuela le llevaban un tiempo rondando. Como recuerda Jaime Abello" : "Además había tenido la fortuna de trabajar para la UNESCO, en el famoso informe MacBride ${ }^{4}$, donde se ha-

1 Noticia de un secuestro, una novela de no ficción, se publicó en 1996. Relata el secuestro varias figuras prominentes de Colombia por parte de los Extraditables de Pablo Escobar ocurrido en 1991.

2 Su contribución a la Misión de sabios, la comisión formada en 1993 por el gobierno del presidente César Gaviria para reflexionar y formular recomendaciones sobre los asuntos de educación, ciencia y desarrollo en Colombia.

3 Jaime Abello es cofundador y director general de la FNPI. Entrevista personal, 4 de junio en Cartagena de Indias, la sede de la FNPI.

4 El Informe MacBride es un documento de la UNESCO publicado en1980 y redactado por una comisión presidida por el irlandés Sean MacBride, ganador del Premio Nobel de la Paz, y cuyo objetivo era analizar los problemas de la comunicación en el mundo y las sociedades modernas. 
blaba de la necesidad de fomentar un periodismo que partiera de la teoría de que hay unos países dominantes y se producían unos flujos de información asimétricos. Gabo no solo tenía una visión global de la época de ese informe, tenía su propia experiencia de su periodismo militante".

La primera manifestación pública del proyecto pedagógico de García Márquez fue un memorable discurso que dio la vuelta al mundo y se convirtió en una suerte de bandera que leen los estudiantes de periodismo en los primero años de carrera: "El mejor oficio del mundo"s. En él dejó plasmados muchos de sus sueños y de sus soluciones casi visionarias: "toda la formación debe estar sustentada en tres pilares maestros: la prioridad de las aptitudes, la certidumbre de que la investigación no es una especialidad del oficio sino que todo el periodismo debe ser investigativo por definición, y la conciencia de que la ética no es una condición ocasional, sino que debe acompañar siempre al periodismo como el zumbido al moscardón".

Una metáfora afortunada para indicar el camino del rigor, de la comprobación, de la claridad por encima de cualquier prisa, de toda primicia. Y ya apuntaba también aquel texto las intenciones, el nombre y el método de su pedagogía: "Un grupo de periodistas independientes estamos tratando de hacerlo desde Cartagena de Indias, con un sistema de talleres experimentales e itinerantes que lleva el nombre nada modesto de Fundación para un Nuevo Periodismo Iberoamericano. Es una experiencia piloto con periodistas nuevos para trabajar sobre una especialidad específica-reportaje, edición, entrevistas de radio y televisión, y tantas otras- bajo la dirección de un veterano del oficio".

El 18 de marzo de 1995 se puede decir que se inició la primera actividad de la FNPI, un seminario sobre libertad de prensa y protección a los periodistas organizado junto con el Committee to Protect Journalists de Nueva York. Allí dijo García Márquez: "temo que alguno de ustedes se pregunte que tiene que ver una escuela de periodismo empírico con los nobles propósitos del comité para protección de los periodistas. Es muy sencillo: un factor esencial en la defensa de la integridad de un periodista es una buena formación profesional".

A petición de Tomás Eloy Martínez y Gabriel García Márquez, Alma Guillermoprieto $^{6}$ dictó el primer taller de la FNPI, entre el 3 y el 8 de abril de 1995, sobre la crónica. Javier Darío Restrepo dirigió, entre el 5 y el 8 de mayo, uno sobre ética periodista. El propio García Márquez uno de reportaje entre el 24 y el 28 de mayo. Tomás Eloy Martínez, uno de edición entre el 30 de mayo y el 3 de junio Phil Bennet hizo el suyo sobre las tendencias de la prensa en los Estados Unidos y Stephen Ferry sobre fotografía. El español Miguel Ángel Bastenier dirigió uno de periodismo internacional, entre el 25 y 27 de octubre.

5 Pronunciado por Gabriel García Márquez en la 52 Asamblea de la Sociedad Interamericana de Prensa, SIP, en Los Angeles, EE.UU, el 7 de octubre de 1996.

6 Alma Guillermoprieto, escritora y profesora mexicana, reportera del diario inglés The Guardian, y después de The Washington Post. Jefe de corresponsales del semanario Newsweek. Desde 1989 escribe sobre América Latina para The New Yorker y The New York Review of Books. 
En septiembre de ese mismo año, 1995, explicó García Márquez su idea de taller: "pensé que la única manera de contar la experiencia era hacerla al estilo de los antiguos maestros del Renacimiento, de persona a persona". ${ }^{7}$

Así fue como el grupo de periodistas comprometidos fueron calculando cómo se financiaría aquella aparente locura y ensayando lo que sería un modelo pedagógico, "que consistía -recuerda Jaime Abello (2012:466)- en promover, desde una institución imparcial e independiente, un proyecto educativo internacional enfocado a la reflexión y la experimentación de laboratorio sobre el oficio".

Jean-Francois Fogel miembro de la junta directiva asegura ${ }^{8}$ que la Fundación nació "como un conjunto de personas que se vincularon a un proyecto para mejorar el periodismo; un periodismo que no cambió durante más de un siglo pero que ahora está trastocado". De modo que además de mejorar, el modelo incluyó la modernización y el adelanto a los nuevos tiempos en el contexto de una época de transición a la convergencia digital.

La maestra que impartió el primer taller, Alma Guillermoprieto, recuerda ${ }^{9}$ el encargo que personalmente le hizo García Márquez: "En cuanto a la forma del taller en sí, tenía pocas ideas concretas, pero sí unas guías claves: serían talleres exclusivamente para periodistas de oficio; se le haría la guerra a la idea misma de la «comunicación social», plaga de las facultades iberoamericanas; y serían talleres prácticos. Y una idea más, que me hizo entender de golpe la sagacidad del personaje: sería impartido por periodistas también, pero no por cualquier periodista: tendrían que ser grandes periodistas, y más allá de eso -aclaró- tener star quality (pronunció con cuidado la frase en inglés). Hay que deslumbrar."

\section{Propósito y metodología}

Este artículo pretende avanzar en la disyuntiva establecida en escuelas, facultades y medios profesionales sobre si el periodismo se enseña o se aprende. Un paso más en la controversia entre los defensores del aprendizaje en la academia y los partidarios de hacerlo en el ejercicio del oficio. $Y$ en último caso, quienes contemplan el periodismo como una profesión universitaria o los que lo ven como una cierta artesanía. Sin entrar en ese enfrentamiento aparentemente irreconciliable sí quiere comprobar si instituciones como la FNPI demuestran que enseñar, y aprender, periodismo de calidad es posible si se parte de un empeño y se tiene un modelo.

Observando in situ su actividad, revisando sus contenidos, repasando sus publicaciones, asistiendo a sus talleres, estudiando los acercamientos teóricos que otras universidades e investigadores han hecho a su modelo, quizá se pueda entender su

7 Frase recogida por la periodista Rosa Mora y publicada en El País, el 7 de septiembre de 1995.

8 Jean Francois Fogel, es periodista y ensayista francés, trabajó para la Agencia France Press, el diario Liberation y el semanal Le Point, asesor del director de Le Monde. Entrevista personal, por Skype, el 18 de junio de 2014.

9 Entrevista personal por correo electrónico, 8 de junio 2014. 
impacto internacional, sus estrategias para conseguir aliados y el futuro del sueño del premio Nobel colombiano. Sobre todo tras su muerte, el 18 de abril de 2014.

La pretensión es comprobar si la actividad de la FNPI, sus talleres, seminarios y encuentros de periodistas responden a un patrón; si sus actividades contribuyen al crecimiento y consideración de una profesión denostada; si es posible acreditar unas líneas ideológicas o estéticas, y descubrir qué asuntos se consideran prioritarios y definidores del modelo.

Con el seguimiento de un caso paradigmático podremos establecer el grado de cumplimiento y también obtener algunos resultados. Se podrá probar la sistematización de actividades y contenidos, si las metodologías de los talleres logran capturar y gestionar el conocimiento que desarrollan. Una exigencia intelectual es testar si junto al compromiso declarado con la ética y la calidad, los retos del conocimiento están relacionados además con la cultura, la ciencia, la tecnología, el estado de derecho, las instituciones democráticas, los derechos humanos, la responsabilidad social y el desarrollo sostenible.

Se va a comprobar el liderazgo, y el impacto real en la comunidad periodística latinoamericana, que ejercen hoy varias decenas de nombres salidos de esos talleres. El análisis de un caso también puede indicar si la pedagogía traspasa la tertulia de café y se asienta en el conocimiento científico y riguroso.

Para realizar este afanoso análisis cualitativo partimos de la participación directa y la observación por tanto cercana de varios de sus talleres. Tomamos uno de ellos, representativo como caso de estudio, el "Taller de Periodismo de Investigación especializado en la cobertura de la seguridad ciudadana", celebrado del 12 al 15 de mayo de 2014 en Bogotá, y cuyos resultados se están viendo hoy, varios meses después.

Al tiempo se repasan las relatorías históricas, se recaba el testimonio de algunos de sus maestros y se realiza el seguimiento de algunas trayectorias profesionales de los alumnos más brillantes.

El estudio de la investigación que hizo la Universidad de los Andes ${ }^{10}$ muestra argumentos, comprobaciones, fortalezas, acciones, expectativas y necesidades. La Universidad de los Andes, en alianza con Radio Nederland Training Centre, estudió entre diciembre de 2006 y febrero de 2007 la realidad de la Fundación, las tendencias en el campo del periodismo y las acciones a emprender para aumentar la efectividad de la misma FNPI.

Junto a esos medidores documentales se realizaron entrevistas personales con su director general, Jaime Abello, y su director gerente, Ricardo Corredor, algunos de sus maestros y miembros de la junta directiva, como Jean Fancois Fogel, Alma Guillermoprieto, Javier Darío Restrepo y Cristian Alarcon. A algunos de alumnos, como Patricia Nieto, Carlos Alberto Giraldo, Carol Pires o Silvana Paternostro.

${ }^{10}$ Estudio que la FNPI encargó a la Universidad de los Andes, Bogotá, en 2006, con la intención de fortalecer su capacidad organizacional y sirvió para diseñar una plan estratégico para el periodo 2008-2012. 


\section{Caso de estudio}

El taller elegido analizar es paradigmático, por un lado y por sus características representa el modelo pedagógico de la fundación y por otro, fue el primero en celebrarse tras la muerte del fundador de la FNPI ${ }^{11}$. El Taller de Periodismo Especializado en la cobertura de seguridad ciudadana lo impartió María Teresa Roderos ${ }^{12}$ y lo tomaron veinte periodistas de Argentina, Bolivia, Perú, Ecuador, Brasil, Venezuela, Colombia, Nicaragua, Costa Rica, El Salvador, Guatemala y México, lo patrocinaba el Banco Interamericano de Desarrollo y el Banco Mundial y se celebró en un conocido hotel de Bogotá. "Esto refleja lo que Gabo esperaba de su fundación -dijo en la presentación Jaime Abello- Convocar periodistas jóvenes y trabajar juntos en lo que denominó la "carpintería del oficio".

Los periodistas tenían entre los 25 y los 30 años y venían de las redacciones de los principales medios, analógicos, o digitales, radios y televisión, de Iberoamérica. Habían llegado al taller, seleccionados por la FNPI, convencidos de la importancia y responsabilidad que suponía aprender periodismo en la Fundación de García Márquez. El requisito, cumplido por todos, era aportar una propuesta de investigación verdadera. La maestra escuchó con atención la exposición de cada una de ellas y se ocupó de analizar, valorar, apuntar pistas, ofrecer salidas aportar soluciones e incorporar ejemplos de sus propias investigaciones. Es decir, armó una de las máximas del proyecto pedagógico: el intercambio de prácticas y experiencias.

Junto a la maestra, dos reporteros experimentados, ex alumnos de la FNPI, aportaron asistencia y consejo, Sebastián Hacher y Óscar Martínez, de Cosecha Roja y El Faro, dos medios prestigiosos de Argentina y El Salvador. En diferentes sesiones el taller auxilió con sus propias fuentes, un director de seguridad ciudadana, un experto en seguridad, un investigador, el Alto Consejero Presidencial para la Convivencia y Seguridad Ciudadana, un ex ministro de educación, que se sometieron a las preguntas de alumnos y maestros.

Ronderos es gran periodista y buena pedagoga. Explicaba aportando experiencias propias. Insistía en la escuela de Gabo con tres ideas: la vocación, la ética y la investigación, "hasta la más mínima nota tiene indagación y búsqueda", como apunta el también maestro Daniel Santoro (2007:17). Y con esas tres llaves sembraba el salón de preguntas que los jóvenes periodistas anotaban: ¿Contribuimos los periodistas a la histeria colectiva, al pánico moral? ¿Ayudamos a instalar el miedo? ¿O estamos criminalizando cosas que no lo son? ¿Magnificamos el papel de los individuos en el crimen y minimizamos el de las instituciones?

Cada mañana se debatían las preguntas, por la tarde se analizaban los proyectos, uno a uno, estudiando la viabilidad. La ficha de cada uno de los veinte periodistas incluía, además de su nombre y el del medio y el país, el título del trabajo, su idea de

${ }^{11}$ Gabriel García Márquez murió el 18 de abril de 2014, menos de un mes antes de la celebración del taller.

${ }^{12}$ Maria Terea Ronderos, Colombia, es directora del Programa Periodismo Independiente de la Open Society Foundations (OSF), la organización filantrópica creada por el multimillonario George Soros, ex directora del sitio especializado en el conflicto armado de Colombia Verdad Abierta y asesora editorial de la Revista Semana. 
reportaje, las fuentes con las que contaría, el alcance geográfico, el tiempo de desarrollo de la investigación, los recursos y los riesgos.

En otros talleres, como en el de Miguel Ángel Bastenier, titulado "Cómo se escribe un periódico", el maestro propone a los alumnos salir a la calle a hacer labores de reportero. El de Alberto Salcedo, "Cómo escribir una crónica", se piensan buenas historias. Todos tienen un alto componente de prácticas reales.

Uno de los recursos pedagógicos que utiliza Ronderos es proyectar una historieta cómica. El hijo dice al padre: quiero dedicarme al crimen organizado. El padre, sin apartar la vista del diario que lee, responde: "sector público o sector privado". Así propone pensar el crimen organizado, como un fenómeno con varias capas que se cruzan, que se confunden. La maestra va soltando lecciones prácticas a cada paso. El suyo es uno de los cientos de talleres que organiza la FNPI en busca del periodismo de calidad. Ella es una "star quality" que orienta y comparte experiencias. Y la veintena de periodistas que escuchan es probable que en un futuro alcancen nombre e impacto.

En este taller se pueden observar las líneas maestras, sistemáticas, del modelo: se proponen ejercicios sobre aspectos del proceso periodístico, como la observación, descripción o el juego de roles; investigación, producción y edición de trabajos; discusión de casos tomados de la realidad; análisis de textos exitosos o fallidos; estudio de los trabajos de otros.

Los proyectos de reportaje que llegaron al encuentro de Bogotá, semielaborados, y que salieron discutidos, enriquecidos y a veces reenfocados fueron: "Cómo se arma la población en Costa Rica"; "Las víctimas no reparadas de Colombia"; "La explotación sexual de niñas en Caracas"; "Cómo cubre el periodismo las protestas de Brasil"; "La ciudad más violenta de Nicaragua"; "Las radios comunitarias de Honduras"; "El microtráfico en el Bronx de Bogotá"; "Las disputas por la droga en la propia policía de Buenos Aires"; "Campesinos guardianes del narco en Bolivia"; "El crimen organizado y el sector de la construcción en Medellín"; "Tregua entre pandillas en El Salvador"; "Los municipios más violentos de Guatemala"; "Los programas de resocialización en Panamá"; "La infancia en el territorio narco de Rosario, Argentina"; "Pulso entre autodefensas, narcos y gobierno en Michoacán, México"; "Las zonas más vulnerables de Ecuador; Las raíces del miedo en Lima".

La organización de un taller como este supone una estructura administrativa, una planificación organizativa y una capacidad para encontrar aliados que financien. Hoy la FNPI es una organización internacional que realiza una actividad económica continuada, independiente y autónoma. Su viabilidad financiera y sus ingresos dependen de alianzas, patrocinios, donaciones y venta de servicios. Una junta directiva de la que forman parte German Rey, Sergio Ramírez, Mercedes Barcha ${ }^{13}$, Jaime García Márquez ${ }^{14}$, María Teresa Ronderos, Jean Francois Fogel, Jon Lee Anderson y Yolanda Pupo de Mogollón, toma las decisiones. Un consejo rector conformado por el colombiano Héctor Abad Faciolince, el norteamericano Jon Lee Anderson, el brasileño Ro-

\footnotetext{
${ }^{13}$ Mercedes Raquel Barcha Pardo es la esposa de Gabriel García Márquez y actualmente ejerce como presidenta de la FNPI.

${ }^{14}$ Jaime García Márquez, hermano del Nobel colombiano.
} 
sental Calmón Alves, el nicaragüense Carlos Fernando Chamorro, el argentino Martín Caparrós, el español Joaquín Estefanía, el puertorriqueño, Héctor Feliciano, el francés Jean-François Fogel, la chilena Mónica González, el nicaragüense Sergio Ramírez y los colombianos Germán Rey y María Teresa Ronderos, propone y sugiere. Un ágil equipo bien relacionado en el plano internacional ejecuta. Este encabezado por su director general y cofundador, Jaime Abello, y una veintena de profesionales, comunicadores sociales en su mayoría, que hacen posible que el sueño de García Márquez se haya convertido en una maquinaria eficiente.

Entre mayo y septiembre, los meses de seguimiento de las actividades de la Fundación para este trabajo, se han producido una cumplida cosecha de talleres, seminarios, conferencias y propuestas pedagógicas. En "Periodismo del nuevo milenio más allá de Internet" se planteó la pregunta de si el afán de desarrollar novedosas aplicaciones multimedia es lo único que la tecnología ha llevado al periodismo. En un coloquio desde Lima se analizó "El poder de la ética en la era del periodismo vigilado". Ha habido un seminario web titulado "Innovación, arte y periodismo", una conferencia sobre innovación y ética periodística a cargo del maestro FNPI Gumersindo Lafuente; un seminario web sobre "Cómo se cubre el dolor", por Marcela Turati, periodista mexicana, experta en la cobertura de tragedias y hechos traumáticos; un encuentro de 30 directores y editores de medios con la Oficina del Alto Comisionado para la Paz con el objetivo de formular recomendaciones para mejorar la cobertura del proceso; un seminario web, "La entrevista con el malvado" sobre los victimarios como fuentes; un taller sobre el uso de herramientas digitales para la investigación periodística; en Río de Janeiro, Brasil, se organizó un Taller de Periodismo de datos en temas ambientales; un Taller de periodismo cultural, en Medellín; un webinar sobre buenas prácticas periodísticas al informar sobre temas de educación; un seminario web sobre los ángulos para abordar la crisis global del agua; un hangout sobre periodismo de datos; un seminario Memeton, o cómo los memes llegaron al periodismo; un Memetón sobre ética y periodismo; el Taller "Cómo se escribe un periódico", impartido por Miguel Ángel Bastenier; un taller de libros periodísticos, dictado por Martín Caparrós.

El formato cambia si es charla, coloquio, seminario, conferencia, encuentro o taller; también varia la duración, una semana, un día, unas horas o un mes; e igualmente puede ser distinto el número de participantes. Lo que permanece inalterable es el patrón: un experto reconocido, un maestro, enseña lo que sabe, muestra su experiencia y lo comparte con un número limitado de seleccionados periodistas.

El modelo tiene la particularidad de retroalimentarse, de apoyarse, de crear redes, alianzas y fortalezas de futuro. Muchos alumnos repiten y algunos acaban convirtiéndose en maestros. El proyecto tiene una capacidad innata, flexible, de amoldarse a los tiempos, a las nuevas tecnologías, a las derivas del periodismo y a que los nuevos maestros ocupen el lugar de los antiguos y conserven el espíritu y el legado de García Márquez.

\section{Los logros}

La labor del taller no acaba cuando termina. El modelo exige un seguimiento del trabajo que deberán continuar los alumnos, una comprobación de los conocimientos ad- 
quiridos por medio de encuestas y una producción de material que forme parte de una biblioteca del conocimiento.

La información de cada taller se sistematiza para enriquecer la labor pedagógica de la FNPI por medio de relatorías, en las que un periodista se encarga de plasmar los conceptos ensayados y experiencias compartidas. Algunos terminan convirtiéndose en manuales. Es el caso de "La invención de la crónica", de Susana Rotker y "Técnicas de investigación", de Daniel Santoro ${ }^{15}$. También se editan antologías o los llamados Cuadernos de periodismo, monográficos sobre temáticas y novedades de los talleres y la profesión. Algunos se convierten en materiales de estudio de otras disciplinas ${ }^{16}$.

Atendiendo a una aproximación cuantitativa el recorrido de cuatro lustros de intercambio de conocimientos y reflexión, arroja resultados irrefutables: decenas de miles de reporteros, editores y directores de medios asistentes a sus convocatorias; varios centenares de seminarios organizados en América Latina y España; una red de periodistas, estudiantes de periodismo, organizaciones de apoyo y facultades de comunicación social que interactúan a través de su plataforma; una producción editorial de varias decenas de títulos impresos, y más de un centenar de publicaciones digitales. Sólo el consultorio en línea sobre ética periodística arroja más de un millar de consultas resueltas. Muestra de su poder de convocatoria son las 1.400 postulaciones de 35 países en la última edición del Premio García Márquez de Periodismo. La FNPI tiene un enorme potencial con su red de periodistas y también con el entramado poderoso de socios estratégicos, patrocinadores y colaboradores conformado por más de 70 organizaciones punteras de América Latina, España, otros países europeos y Estados Unidos.

Junto al análisis cuantitativo que arroja números contundentes aparece otro cualitativo lleno de leyendas del periodismo: el propio García Márquez, Ryszard Kapuscinski, de Tomás Eloy Martínez, Germán Rey, Alma Guillermoprieto, Stephen Ferry, Rosental Calmon Alves, Phil Bennet, Javier Darío Restrepo, Jon Lee Anderson, Daniel Santoro, Jean-François Fogel, Francisco Goldman... y nombres que hoy están a la cabeza de medios importantes, son admirados y premiados, y asistieron a talleres de la FNPI: Alejandro Almazán, Óscar Martínez, Juanita León, Abad Faciolince, Marcela Turati, Martín Caparrós, Boris Muñoz, Luz María Sierra, Patricia Nieto... Hay pocos periodistas con nombre, directores o fundadores de medios en América Latina que no hayan pasado por la FNPI.

Tras viene años, el sueño creció, se desarrolló en modelo de negocio y hoy es una gran red global que tiene actividades relacionadas con el periodismo por todo el mundo: consultorios, seminarios, intercambios con universidades y fundaciones... Jaime Abello recuerda unas palabras de García Márquez en 2006. Repasaban los lo-

${ }^{15}$ Ambos libros, publicados por la FNPI en colaboración con el Fondo de Cultura Económica, que se han convertido en manuales de periodismo, salieron de las salieron de los talleres que dictaron la venezolana Susana Rotker y el argentino Daniel Santoro.

16 "La otra cara de la libertad. La responsabilidad social empresarial en medios de comunicación de América Latina" es un ejemplo. El material de este libro es producto de las entrevistas, documentos y casos que aportaron los medios de comunicación de América Latina participantes en el estudio. 
gros de su modelo, el impacto, los aliados, los talleres: "Y pensar que esto estaba en nuestra imaginación".

Se pueden distinguir cuatro etapas en la historia de la FNPI. La primera, entre 1995 y 2000 , coincide con el desarrollo de la idea, su puesta en práctica y pasión de García Márquez en lo que llamaba los talleres de las cinco de la tarde. La segunda, entre 2000 y 2006 es la del asentamiento del modelo de negocio. Es un tiempo de búsqueda de aliados y socios, de planes de expansión, y también de dudas. La tercera entre 2007 y 2013, coincide con la sustitución de los grandes maestros, algunos problemas económicos Se establece por eso un Plan estratégico de cinco años. La cuarta se precipita por la muerte del fundador, en abril de 2013. La máquina parece engrasada y se hace una fuerte apuesta por el premio García Márquez de Periodismo.

Abello ve el futuro con optimismo: "ya hicimos una reflexión a los diez años de la fundación, en 2005, sobre el futuro del periodismo y llegamos a la conclusión de que teníamos que centrarnos en buscar una adaptación". Eso les llevó a replantear el ideal de la fundación: "llegar a la gente con más talento, más liderazgo, que de manera natural se convierten en multiplicadores".

Gabriel García Márquez fue capaz de buscar y encontrar aliados que creyeran en sus sueños y los financiaran. "Hay un sector llamémoslo profesional al que pertenecemos que a nivel mundial se llama Medias Development (desarrollo de medios), y es un conjunto de instituciones que tienen como misión principal fomentar el que exista y prospere el periodismo independiente", explica Jaime Abello. Se trata de un sector con una historia muy ligada "a una visión estratégica de los países del norte de los EEUU y de los países europeos, con instituciones que apoyaban la transición política y el periodismos libre, y la libertad de expresión".

La descripción principal del estudio de la Universidad de los Andes (2006) puede servir de carta de presentación para la definición de la Fundación: Es independiente, innovadora, inseparable del nombre de García Márquez, económicamente sólida, líder en sus campos de actuación, y está a la vanguardia en la formación de periodistas. Afirma en su trabajo que es reconocible porque "ofrece programas diferenciados y de excelencia, con alto impacto en los periodistas y en los medios de comunicación".

\section{Discusión}

Aun con el reconocimiento del periodismo internacional, la FNPI ha arrastrado a lo largo de su historia una cierta demonización, un relativo desprecio académico, que le ha servido en ocasiones para fortalecerse y a veces para sembrar alguna duda sobre su modelo.

Una parte de la sociedad académica ve con reparos su actividad, básicamente práctica, y desconfía de su acreditación. Los propios miembros del Consejo Rector, en permanente debate, no logran acordar una estrategia para alcanzar al mismo tiempo entendimiento y reconocimiento. En algunos de sus debates, como el celebrado para aprobar el plan director 2008-2012, mientras Jaime Abello resaltaba cómo en la práctica las universidades sí han sido aliadas de la FNPI, Javier Darío Restrepo expresaba la necesidad de que la FNPI aportara su experiencia en el rediseño de los programas académicos de las facultades de Comunicación Social. Pero Jean Francois Fogel advertía del peligro de asociar la "marca" de la fundación con facultades de comunica- 
ción y Héctor Feliciano por su parte describía la relación de la FNPI con las universidades como no sistemática.

En el estudio de la Universidad de los Andes se indica: "Al no estar vinculada a una universidad y, por tanto, no tener una certificación académica, RNTC concluyó que necesitaba una certificación en calidad" ${ }^{17}$. La explicación fue que su reputación podía no ser suficiente para conseguir fondos.

El estudio de la universidad de los Andes detectó en 2007 el impacto, las carencias y las oportunidades de la FNPI, aun visibles hoy día. Discurría que existe la percepción de que la FNPI es una formación "elitista" a la que no se accede fácilmente. La imagen que se asocia a la FNPI es la de "capacitación para periodistas con muy alto vuelo" y que los talleres producen una expectativa que luego no pueden incorporarse al trabajo diario de la fundación. Al finalizar un taller se abren posibilidades de intercambio, proyectos conjuntos, que luego quedan en el aire, "la FNPI ya está trabajando en su próximo taller". Observando el ritmo de trabajo, de viajes, de actividades que se solapan, da la sensación de maquinaria imparable que necesita el movimiento continuo.

Indica también el estudio, y lo ha comprobado este investigador, que la FNPI dedica todos sus esfuerzos a la formación de periodistas, pero hay un cierto descuido en la sistematización del saber que produce. La obtención de conocimientos, de experiencias periodísticas de calidad es ingente, sin embargo no termina en una biblioteca activa y disponible para estudiosos. Demasiados informes, relatorías, videos y conferencias perdidos entre los diferentes formatos y la falta de tiempo para ordenar, categorizar y codificar. Hay en la web cuidada, y entre las paredes de la casa de la calle San Juan de Dios, un acervo cultural y periodístico impagable pero diseminado y por catalogar. Se observa que está por hacer una buena gestión del conocimiento. Maestros y directivos reconocen cierto desaprovechamiento de los materiales que se han generado, la falta de capacidad para administrar los flujos de información y la carencia de canales para difundirlos.

En el Plan Estratégico Multianual 2008-2012 se decía que el modelo pedagógico de la Fundación es el pilar de todas sus acciones y constituye su principal factor de diferenciación. Se recomendaba potenciarlo y se insistía en que los talleres constituyen el elemento básico de la propuesta pedagógica por lo que se aconsejaba que representaran al menos el 50 por ciento de sus actividades. Sin embargo en la actualidad, los Tuitdebate, Webinar, Twitcam, charlas on line, conferencias, coloquios, debates y encuentros, ocupan más que ese $50 \%$ deseable. Algunos maestros y alumnos temen que se pierda el espíritu del taller, lo que constituye el pilar del modelo.

Otro elemento de discusión es la trascendencia real de enseñanzas y aprendizajes. La FNPI selecciona a los mejores periodistas jóvenes y en sus talleres les proporciona una preparación de muy alto nivel, siempre sobre la buena narración, la investigación rigurosa y la ética. Los programas brindan oportunidades de cambio y mejora a pe-

${ }^{17}$ RNTC, Radio Nederland Training Centre, Programa Holandés para el Fortalecimiento Institucional de la Educación y Capacitación Postsecundaria (NPT) de la Organización Holandesa de Cooperación Internacional en Educación Superior (Nuffic) institución que colaboró en el estudio de viabilidad. 
riodistas individuales pero la duda es si logra promover un cambio en los medios a los que pertenecen los periodistas.

\section{Conclusión}

La FNPI es hoy un centro dinámico y de excelencia para el aprendizaje del periodismo de calidad. El modelo pedagógico desarrollado y aplicado por los mejores periodistas ha logrado que la Fundación inventada por la visión de García Márquez se haya convertido en una cantera de reporteros de primer nivel. Muchos han fundado sus propios medios punteros y algunos han logrado premios y reconocimientos de modo que son buscados y fichados por los grandes medios.

El mérito del centro es haber sabido encontrar el talento, hacerlo crecer con la práctica y el intercambio y facilitar sus interrelaciones de modo que se formen redes potentes.

Eso fue lo que vivieron los veinte jóvenes periodistas una semana de mayo en Bogotá. Compartieron las vivencias de la maestra María Teresa Ronderos, aprendieron a investigar y desarrollaron sus propias propuestas. Entre todos buscaron respuestas porque la maestra insistió en que una de las claves del buen periodismo son las preguntas, el no conformarse. Al final, el taller y sus patrocinadores, el Banco Mundial, decidieron repartir entre los talleristas un dinero que les ayudara en su investigación. Entre todos votaron las ideas de reportaje más atractivas, más periodísticas. Las cinco primeras recibieron 1000 dólares cada una. Cinco, ochocientos dólares, y el resto 500 dólares.

Desde mayo a septiembre, cada uno de los veinte periodistas ha trabajado en su medio y ha investigado su historia. Por correo electrónico, web o Skype tanto María Teresa Ronderos como Oscar Martínez y Sebastián Hacher, así como el equipo de la FNPI fueron resolviendo dudas, dando ánimos, proponiendo nuevos caminos.

Hoy aquellas propuestas se han convertido en reportajes y crónicas que acaban de publicar La Nación, de Costa Rica, El País, de Cali, El Nacional, de Caracas, Jornal da Tarde, de Sao Paulo, El Nuevo Diario, de Managua, Radio Progreso, de Honduras, Caracol Radio, de Bogotá, Clarín, de Buenos Aires, El Deber, de Sana Cruz de la Sierra, en Bolivia, El Espectador, de Bogota, El Colombiano, de Medellín, La prensa Gráfica, de El Salvador, Plaza Pública, de Guatemala, La Prensa, de Panamá, El Ciudadano, de Rosario, en Argentina, El País, de México, Ecuavista, de Guayaquil, El Diario Liberal del Sur, de Veracruz y en América Television, de Lima.

Todos parecen haber entendido el método que resume Daniel Santoro (2004:13): "trabajo intensivo durante una semana, tertulias profundas, intercambio de experiencias, mucha documentación y agilidad intelectual para no estancarse en debates sobre definiciones academicistas".

Eso significa que se aprende, como indican Joaquín Estefanía, Jaime Abello, Jean Francois Gogel o Javier Darío Restrepo. Los maestros comparten, los alumnos se fijan, imitan y repiten. El método, entendido como el conjunto articulado de los principios y estrategias a través del cual se transmite y se recibe el conocimiento sobre el periodismo habla de aprender, pero está tan ajustado, tan diseñado que enseña.

Todos los periodistas vocacionales y seleccionados que llegan a la FNPI han completado la licenciatura o los estudios de grado en una universidad prestigiosa y están 
contratados por medios de comunicación, si bien los editores de esos medios están poco dispuestos a financiar la capacitación de sus reporteros, lo que hace que la fundación deba buscar becas y aliados.

La FNPI no es universidad, no es escuela pero ofrece el rigor y proporciona el prestigio que anunciaba su fundador en el discurso que leyó en 1996, cuando dijo que el periodismo era el mejor oficio del mundo y se propuso crear las condiciones ideales para aprenderlo, seguramente con la secreta aspiración de enseñarlo, porque en su visión mostró lo que le faltaba ya entonces y le sobraba a la profesión: "el oficio no logró evolucionar a la misma velocidad que sus instrumentos, y los periodistas se extraviaron en el laberinto de una tecnología disparada sin control hacia el futuro".

$\mathrm{Su}$ denuncia iba dirigida a la dejación, a la entrega del periodismo a los poderes políticos y económicos, a los dictados de la publicidad y al abandono de su misión como servicio ciudadano. Un círculo perverso que ha llevado a muchos medios a no hablar de cosas que puedan molestar a sus anunciantes o sus benefactores. García Márquez llego a hablar en aquel discurso de "operación rescate". Es lo que se puede observar que ha hecho su "criatura": no enseñar a ser periodistas, sino mejorar con la práctica a los que ya lo son.

La clave del éxito del modelo acaso está en el número de alumnos y la aspiración irrenunciable a la calidad. En cuanto al primero recuerda Alama Guillermoprieto: "Peleé durante quince años y hasta el último taller por mantener un grupo de doce, que es el máximo que permite la gran cantidad de atención individual a cada tallerista". No cabe por tanto en el modelo pedagógico de la FNPI ni la masificación, ni el olvido de las reglas de servicio público, ni la falta de ética, ni la búsqueda de la primicia a cualquier precio, ni escritura mediocre.

Se puede hablar por tanto de un medido modelo que enseña sin aulas y sin exámenes pero no deja nada al azar. Ni la selección de los maestros, ni la elección de los talleristas. Aquellos entre los grandes, los otros elegidos por su talento. Una norma tan sencilla. Los más dispuestos aprenden de los mejores en las condiciones más favorables. Porque los encuentres se producen en un lugar confortable todas las herramientas de la tecnología y la documentación a su alcance.

Los resultados son fácilmente visibles: aprenden rápido, se entusiasman, empiezan a destacar y conformar redes de apoyo y retroalimentación. Y como acercamiento cualitativo, más de 400 talleres y seminarios por los que han pasado más de 10.000 periodistas que consolidan a la FNPI como uno de los centros de formación y desarrollo profesional de periodistas más prestigiosos del mundo. Donde el periodismo, si no se enseña, sí se aprende.

\section{Referencias bibliográficas}

BASTENIER, Miguel Ángel (2009): Cómo se escribe un periódico. México, Fondo de cultura económica, FNPI.

GARCÍA MÁRQUEZ, Gabriel (1999): "Ilusiones para el siglo XXI", Conferencia, sesión inaugural del Foro América Latina y el Caribe antes el Nuevo Milenio. París, 8 junio de 1999. 
GARCÍA MÁRQUEZ, Gabriel (1996): Discurso "El mejor oficio del mundo". $52^{a}$ asamblea de la Sociedad Interamericana de Prensa, SIP. Los Angeles, U.S.A., 7 de octubre de 1996.

GARCÍA MÁRQUEZ, Gabriel (1995a): "Discurso inaugural de la FNPI", antes el Committee to Protect Journalists de Nueva York (CPJ). Cartagena de Indias, Colombia, el 18 de marzo de 1995.

GARCÍA MÁRQUEZ, Gabriel (1995b): "Manual para ser niño". Tomo 2 de la colección Documentos de la Misión, Ciencia, Educación y Desarrollo: Educación para el Desarrollo. Presidencia de la República - Consejería para el Desarrollo Institucional - Colciencias) Santafé de Bogotá D.C.

GARCÍA MÁRQUEZ, Gabriel (1994): "Por un país al alcance de los niños". Ceremonia de entrega del Informe de la Misión de Ciencia, Educación y Desarrollo, El Espectador. Bogotá, 23 de julio de1994.

KAPUSCINSKI, Ryszard (2002a): Los cinco sentidos del periodista, Estar, ver, oír, compartir, pensar. México, Fondo de cultura económica.

KAPUSCINSKI, Ryszard (2002b): Los cínicos no sirven para este oficio. México, Fondo de cultura económica.

MARTÍNEZ, Tomás Eloy (1997): "Periodismo y narración: desafíos para el siglo XXI". Conferencia pronunciada ante la asamblea de la SIP el 26 de octubre de 1997, en Guadalajara, México.

MARTÍNEZ, Tomás Eloy (1996): "Defensa de la utopía". Discurso ofrecido en el Taller-Seminario Situaciones de crisis en medios impresos, dictado en Santa Fe de Bogotá del 11 al 15 de marzo de 1996.

RESTREPO, Javier Darío (2004): "El zumbido y el moscardón". Taller y consultorio de ética periodística. México, Fondo de cultura económica.

REY, Germán (coord., 2008): La otra cara de la libertad, La responsabilidad social empresarial en medios de comunicación de América Latina. Bogotá, NNPI.

RONDEROS, Maria Teresa y PARRA, Óscar (2007): Guías para periodista: Cómo informar sobre la educación. Cartagena de Indias, Colombia, FNPI.

ROTKER, Susana (2006): La invención de la crónica. México, Fondo de cultura económica.

SANTORO, Daniel (2004): Técnicas de investigación. Métodos desarrollados en diarios y revistas de América Latina. México, FNPI, Fondo de cultura económica.

VV.AA. (2012): Gabo periodista. Antología de textos periodísticos de Gabriel García Márquez. Bogotá, Colombia, Editorial Maremagnum.

VV.AA. (2005): "Hacia dónde va el periodismo, responden los maestros". Memoria de la Conferencia organizada por la FNPI y CAF. Bogotá, Colombia, 28 de junio.

VV.AA. (2003): "Ética, calidad y empresa periodística en América Latina". Memoria del Seminario realizado en Monterrey, México, 1-28 de septiembre. 